\title{
Skull Evolution in the Rhinocerotidae (Mammalia, Perissodactyla): Cartesian Transformations and Functional Interpretations
}

\author{
Gerald S. Bales ${ }^{1,2}$
}

\begin{abstract}
Cartesian transformation, applied as a landmark morphometric method, is used to investigate some of the evolutionary shape changes leading to the skulls of the modern rhinoceroses. The early Oligocene genus Subhyracodon serves as the primitive shape from which the extant genera (Dicerorhinus, Rhinoceros, Diceros, and Ceratotherium) have been transformed. Coordinate data for 21 landmarks, defined in lateral view, are analyzed by the computer program Thinplate Splines. Each of the four transformations are interpreted separately as shape change from Subhyracodon. Computed results for Rhinoceros are also compared with previous results obtained by visual interpretation (the classic method). Among the extant genera, Ceratotherium and Rhinoceros have the most derived shapes and are opposites with respect to orientation of the occiput and relative size of the mandible angle. The significance of these foci of change is discussed in terms of the functions of the masseter and posterior temporalis muscles. In head positions associated with feeding on short vs. tall grasses, the two skull shapes are consistent with a role for these muscles in support of a large mandible against gravity. This common factor may help to explain both shapes.
\end{abstract}

KEY WORDS: Cartesian; transformation; splines; thinoceros; skull; evolution.

\section{INTRODUCTION}

The four extant genera of Rhinocerotidae (Dicerorhinus, Rhinoceros, Diceros, and Ceratotherium) represent endpoints of a long evolutionary history in the Cenozoic (Prothero et al., 1989). Rhinocerotids (rhinoceroses) arose in the Eocene as one of three familial clades comprising a superfamily (Rhinocerotoidea), and their phylogeny is recorded by one of the largest fossil collections available for large vertebrates. Recent phylogenetic studies (Prothero et al., 1986) have recognized at least 26 genera (Fig. 1). The early Oligocene genus Subhyracodon is representative of the primitive rhinoceros skull condition. This long phylogeny with a well-characterized primitive taxon allows the study of shape evolution leading to the modern taxa.

One of the first attempts to characterize rhinoceros skull evolution in general terms was by Thompson $(1917$, p. 761). He included rhinoceros skulls as an example in a chapter on "comparison of related forms" where he introduced and formalized Cartesian

\footnotetext{
${ }^{1}$ Department of Anatomy, Western University of Health Sciences, 309 E. Second Street College Plaza, Pomona, California 91766-1889.

${ }^{2}$ To whom correspondence should be addressed.
} 


\section{RHINOCEROTOIDEA}

Hyrachus

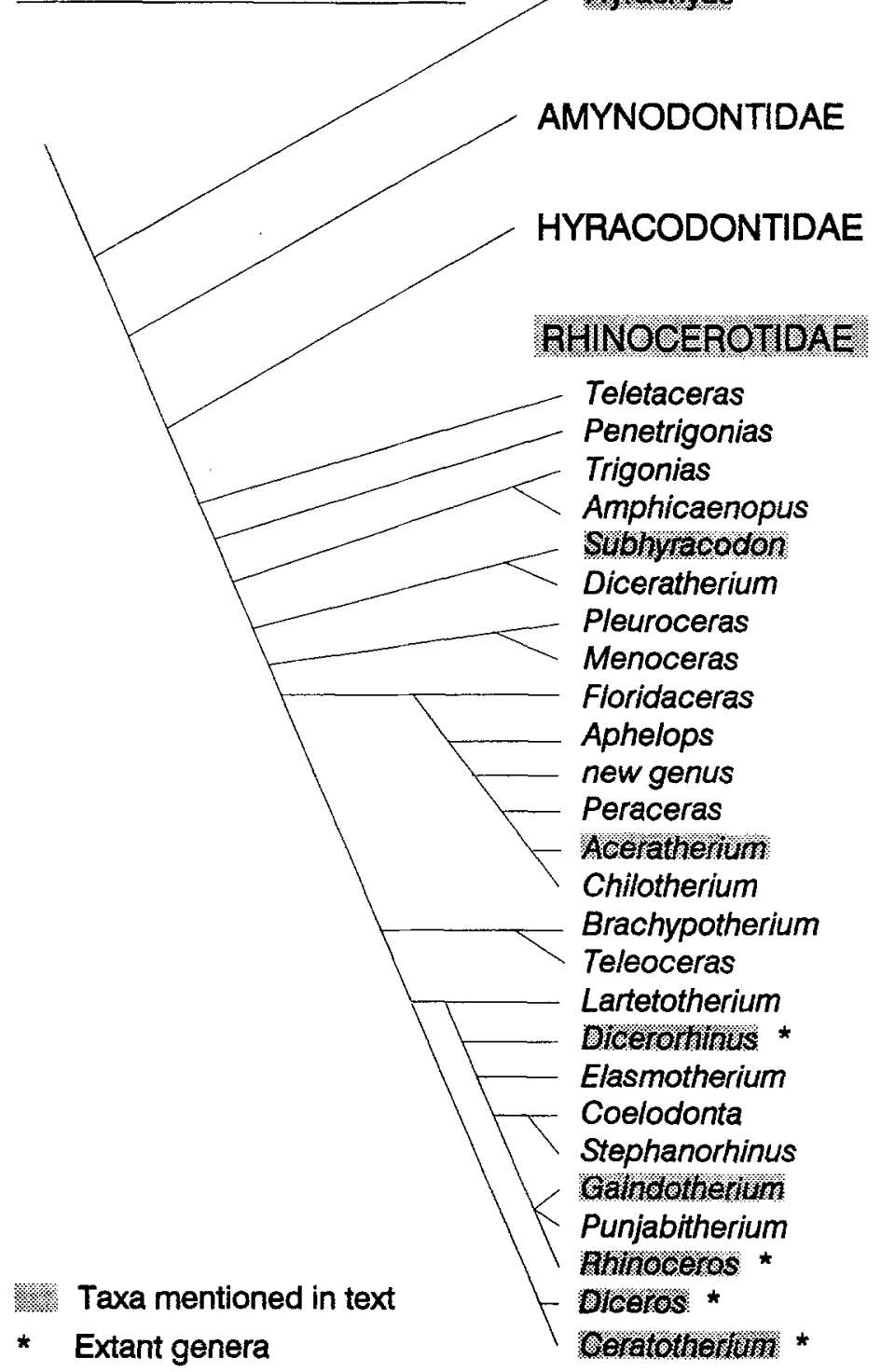

Fig. 1. Phylogeny of the Rhinocerotoidea showing genera of the Rhinocerotidae (after Prothero et al., 1986). Shaded taxa are those mentioned in the text. Starred (*) taxa are extant.

transformation. The idea that evolutionary changes between closely related taxa could be summarized by geometric constructs was demonstrated by Thompson using a variety of animal and plant examples. Thompson's analyses of transformation in fishes were particularly elegant and convincing. In the rhinoceros example (Fig. 2), he examined the transformation from Hyrachyus (sister taxon of the three rhinocertoid families) to 
a.

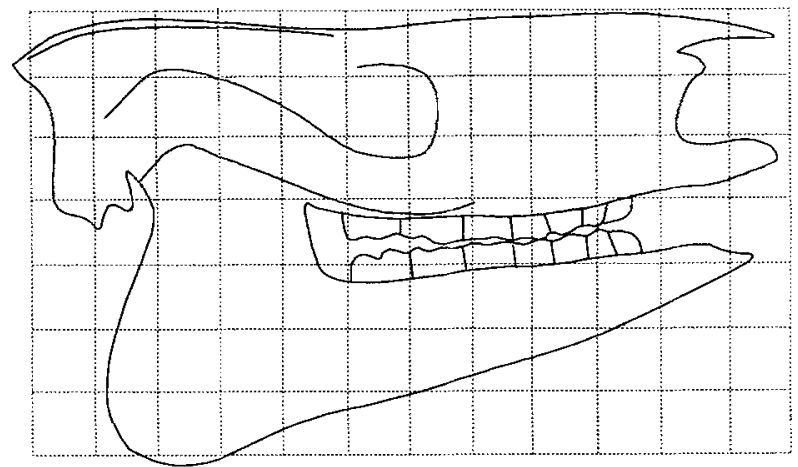

b.

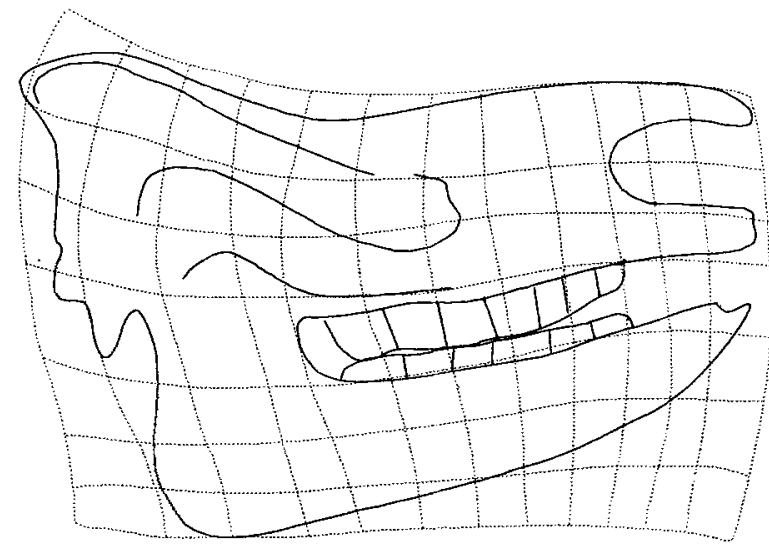

Fig. 2. Cartesian transformation of Hyrachyus to Aceratherium after Thompson (1917, p. 761). (a) Hyrachyus with superimposed, arbitrary rectangular (orthogonal) reference grid. (b) Aceratherium with grid lines deformed so as to pass across homologous points or areas, relative to Hyrachyus.

Aceratherium, a Miocene rhinocerotid. Thompson concluded that the shape changes observed (an anterior-posterior "double curvature" and a dorsal-ventral "expansion') would produce the skull of an existing rhinoceros when extrapolated.

In a more advanced analysis, Colbert $(1935$, p. 190) used the Cartesian approach to demonstrate the intermediate position of Gaindatherium between Subhyracodon and Rhinoceros. He concluded that Rhinoceros is an extrapolation ("exaggerated accentuation') of Gaindatherium and is characterized by greater depth relative to length. Dicerorhinus was also included for comparison: Colbert pointed out that this skull has cheek teeth more forward in position and lacks a "strong forward inclination of the occiput." Colbert's transformation from Subhyracodon to Rhinoceros (Fig. 5) is discussed further below in comparison with computed results.

Neither Thompson nor Colbert analyzed their transformations in detail or discussed 
any functional consequences of the shape changes. The classical method used by both (here called Thompsonian transformation, TT) involves manual construction of a reference grid of orthogonal lines over the primitive (untransformed) shape. Corresponding lines, curved as needed, are then drawn on the evolved (transformed) shape such that they pass across comparable areas or through homologous points (landmarks). The overall effect is that of a deformation of the reference grid. The shape differences between primitive and derived taxa represent evolutionary changes in position and proportion of homologous structures. These structural changes in most cases will be accompanied by changes in the relative positions of landmarks. The shifts in landmark positions therefore contain information about the transformation. Recent advances in landmark morphometric methods have resulted in the Thin-plate Splines program (TPS), a computer implementation of the Cartesian method, but based on coordinate data for landmarks (Bookstein, 1989, 1990, 1992; Rohlf and Bookstein, 1990). Currently, the only available software for this procedure is that written by Rohlf (1990b). He continues to improve and expand his programs, including the addition of Windows versions, which are available through SUNY Stoneybrook WEB sites (http://life.bio.sunysb.edu).

The purpose of this study is to (1) generate landmark-based Cartesian transformation grids for the skulls of each of the extant rhinoceros genera in the context of their evolution from Subhyracodon, and (2) to biologically interpret the evolved shape differences in terms of skull functions.

\section{MATERIALS AND METHODS}

\section{Thin-plate Splines}

The method of Thin-plate Splines (TPS) was used to generate Cartesian transformation grids on a DOS 386 IBM PS2 microcomputer. The TPS method (Fig. 3a) uses $x, y$ coordinate pair data derived from homologous landmarks on two different specimens (shapes). Each shape is represented by a matrix of $x, y$ values listed in the same order. The order of operations on the data is as follows. First, TPS mathematically translates, rotates, and isometrically scales the "shapes" (datasets) to obtain the closest fit of the landmarks prior to deformation. This is a procrustes approach using a least squares criterion. Second, it analytically determines the deformations (in the form of interpolation functions) required to directly superimpose homologous landmarks from one shape on to the other. Last, the analytical functions are used to construct a grid representing the deformation(s). This deformation grid is the Cartesian transformation grid. An orthogonal reference grid, fundamental to the classical method, is not required. For more detailed analyses, TPS can factor the transformation grid into one affine and multiple nonaffine components. In relation to theoretically possible types of transformation (Fig. $3 b$ ), the affine component (as implemented by TPS) is a uniform global transformation. It is a special case in which all initially parallel lines remain parallel after transformation. The nonaffine components are generally local and nonuniform. It should be noted that, in terms of evolution, transformation grids represent net change between the primitive and derived forms. They do not necessarily provide information about the intervening pathway of change. 

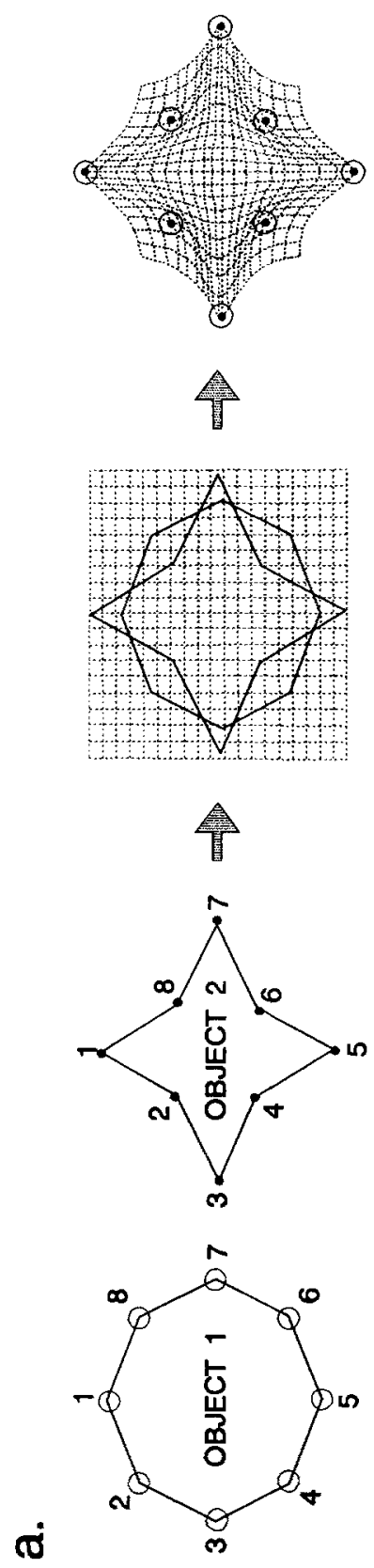

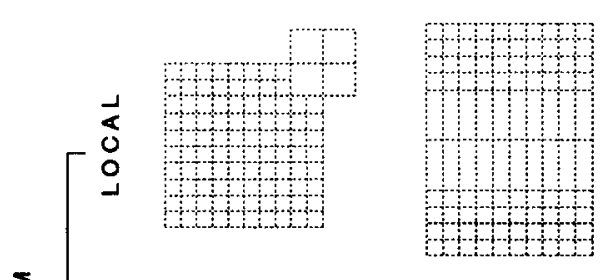

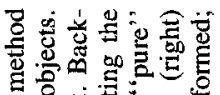

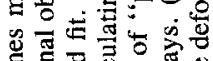

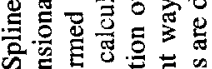
के

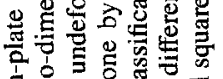

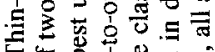

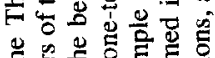

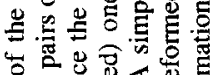

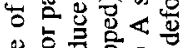

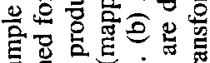

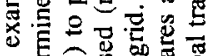
可

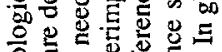
응

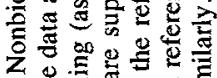
ब讨 .

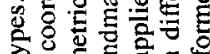

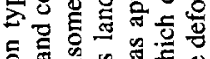
ᄃ

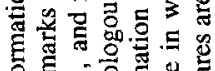

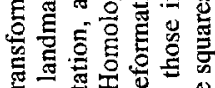

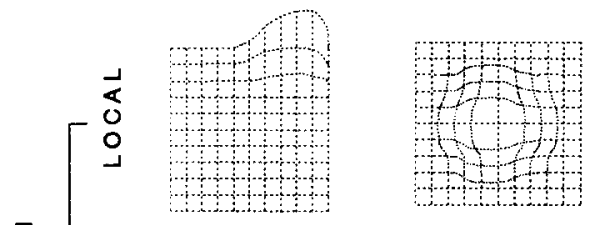

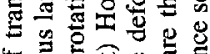

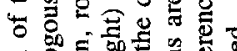

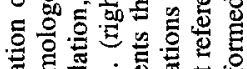

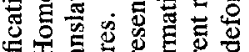

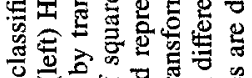

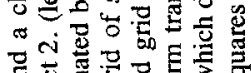

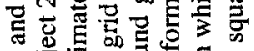

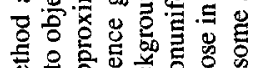

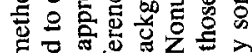
总跑的司志

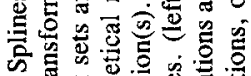
क

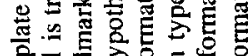

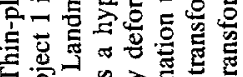

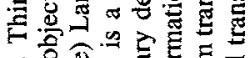

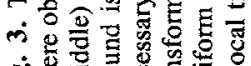
분 


\section{Rhinoceros Landmarks}

The primary data consist of line drawings of Subhyracodon (S. occidentalis) and each of the extant genera (see Figs. 5-7). Outline drawings of the five genera were derived from two sources. Drawings of Subhyracodon, Dicerorhinus, and Rhinoceros (R. unicornis) were adapted from Simpson et al. (1960), who reproduced Colbert's (1935) figures. These outlines were used to maximize comparability between Colbert's TT grid and the TPS grid. The drawings for Diceros and Ceratotherium were made by projection of photographs of representative specimens taken by the author. The visual perspective for comparison throughout is a lateral view with posterior on the left. Twenty-one landmarks were defined to capture shape information from the line drawings (Table I and Fig. 4). Thirteen landmarks may be considered "true" landmarks in that they are defined directly by morphological features. Eight landmarks are constructed by reference to the morphological landmarks. The constructed landmarks occur on the margin of the skull and are necessary to characterize shape details along a continuous curvilinear feature. Twelve of the 21 landmarks are peripheral and capture overall shape while nine landmarks capture internal detail, especially in the region of the tooth rows. Because the cheek teeth have not significantly changed and they define five of the internal landmarks, their influence may be weighted in determining the final transformation. That is, they may function as an area of "anchoring" in terms of the initial relative positions of the remaining landmarks before deformation.

Table I. Definitions of Landmarks Used for Thin-plate Spline Analysis of Rhinoceros Skull Outlines ${ }^{a}$

\footnotetext{
Morphological landmarks

1. Anteriormost point on the nasal bone (nasal tips).

2. Posteriormost point on the margin of the occipital crest.

3. Posteriormost point on the occipital condyle.

4. Superiormost point on the upper margin of the zygomatic arch.

5. Anteriormost point on the margin of the orbit.

6. Posteriormost point on the margin of the nasal notch.

7. Anteriormost point on the premaxilla.

8. Anterior margin of the second upper premolar at the alveolus.

9. Anterior margin of the first upper molar at the alveolus.

10. Posterior margin of the third upper molar at the alveolus.

11. Posterior margin of the third lower molar at the alevolus.

12. Anterior margin of the first lower molar at the alveolus.

13. Anteriormost point on the inferior margin of the mandible.
}

\section{Constructed landmarks}

14. Inferior margin of the nasals on the perpendicular bisector of 1 and 6 .

15. Superior margin of the nasals on the vertical line through 14 .

16. Posterior margin of mandible in the occlusal plane of the tooth row.

17. Inferior margin of mandible on vertical line midway between 10 and 16 .

18. Margin of the mandible on the perpendicular bisector of 16 and 17 .

19. Inferior margin of the mandible on the vertical line through 12 .

20. Dorsal margin of the skull on the vertical line above 9.

21. Dorsal margin of the skull on the vertical line above 10 .

${ }^{a}$ Morphological landmarks are defined by specific morphological loci. Constructed landmarks are defined by reference to morphological landmarks. 


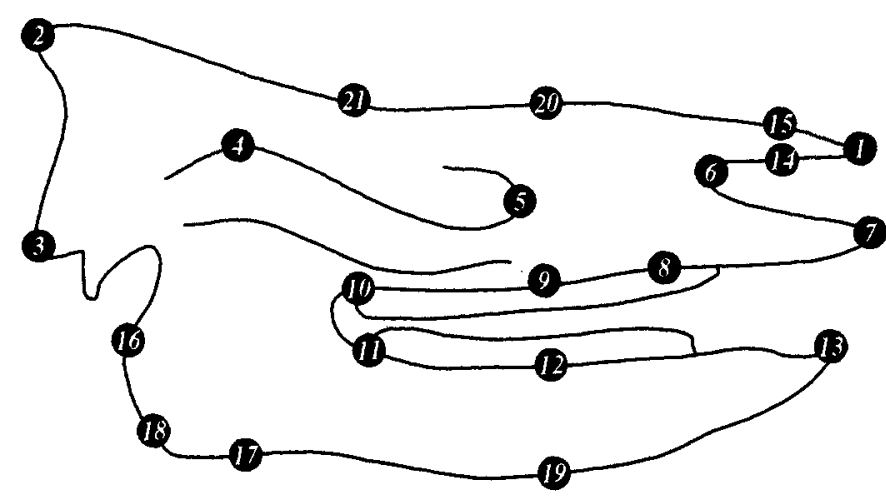

Fig. 4. Positions of landmarks used for Thin-plate Spline analysis. Landmarks are defined in Table I and shown on the outline of Subhyracodon.

Landmark coordinates were obtained from a Summagraphics Summasketch III tablet using DS-Digit software (Slice, 1990). Grids were generated by TPS and captured from the screen by Pizzaz Plus (1990). Pizzaz output was digitized into Freelance Graphics (Lotus, 1993) to produce the figures.

\section{RESULTS}

\section{TT (Manual) and TPS (Computed) Grids}

A comparison of Colbert's (1935) transformation from Subhyracodon to Rhinoceros with that of the TPS transformation is shown in Fig. 5. Features of the shape change shown by Colbert's grid (Fig. 5a, bottom) include (1) forward displacement of the occiput, (2) elevation of the nasal region, (3) relative broadening of the skull posteriorly, (4) shortening of the area between the orbit and nasal notch, and (5) a relative deepening of the skull overall (brachycephaly). The first two of these changes taken together produce a slight dorsal saddling effect in the grid. Generally, the grid seems to adequately reflect the major differences observed when the two skulls are inspected, especially the broadness of the posterior aspect of Rhinoceros relative to the anterior facial region. The TPS grid (Fig. 5b, bottom), representing the total deformation, shows both similarities and differences with Colbert's grid. Forward displacement of the occiput is well shown and is visually enhanced by the "compression"' of the vertical lines through that region (as if the bone was squeezed to accommodate the displacement). Elevation of the nasal region is also indicated but in a somewhat different way such that the entire section of the grid is moved up and forward. Saddling is more evident within the grid than along the upper margin. Shortening of the distance between the orbit and nasal notch is also indicated by the smaller distances between vertical grid lines, and there seems to be a general counterclockwise rotation in this region. The TPS grid does not adequately reflect the posterior broadening or the overall relative deepening of the skull. Where Colbert (1935) shows dorsoventrally-elongated grid cells in the region of the angle of the mandible, the TPS shows paradoxically flattened grid cells. This might be explained by the 


\section{CARTESIAN TRANSFORMATION METHODS}

a.
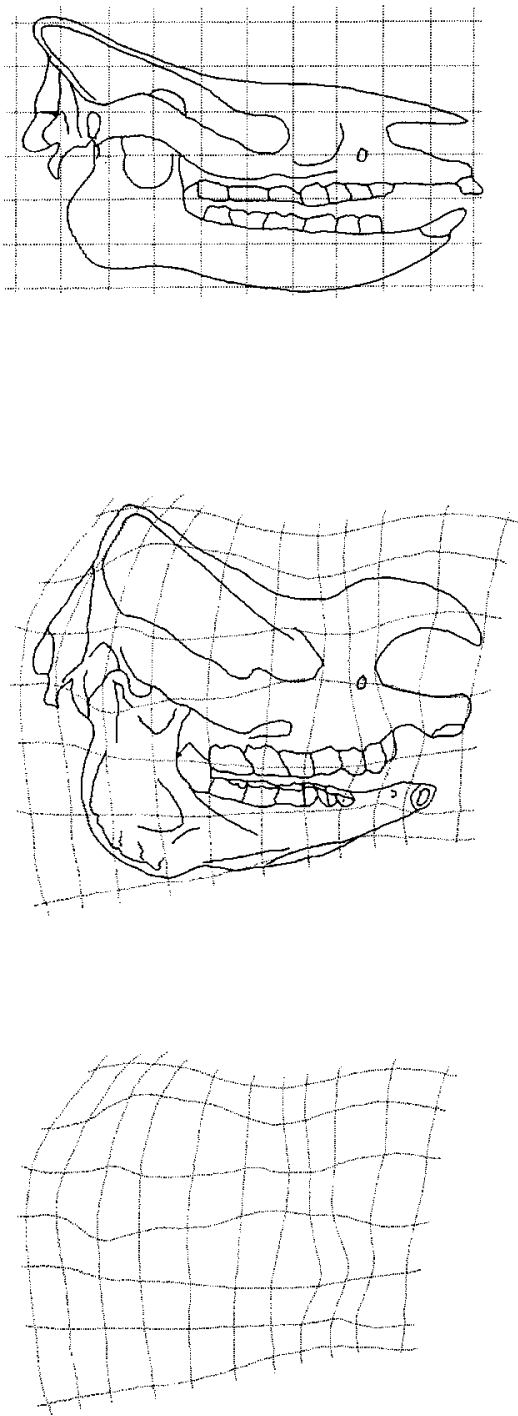

b. Thin-plate Splines
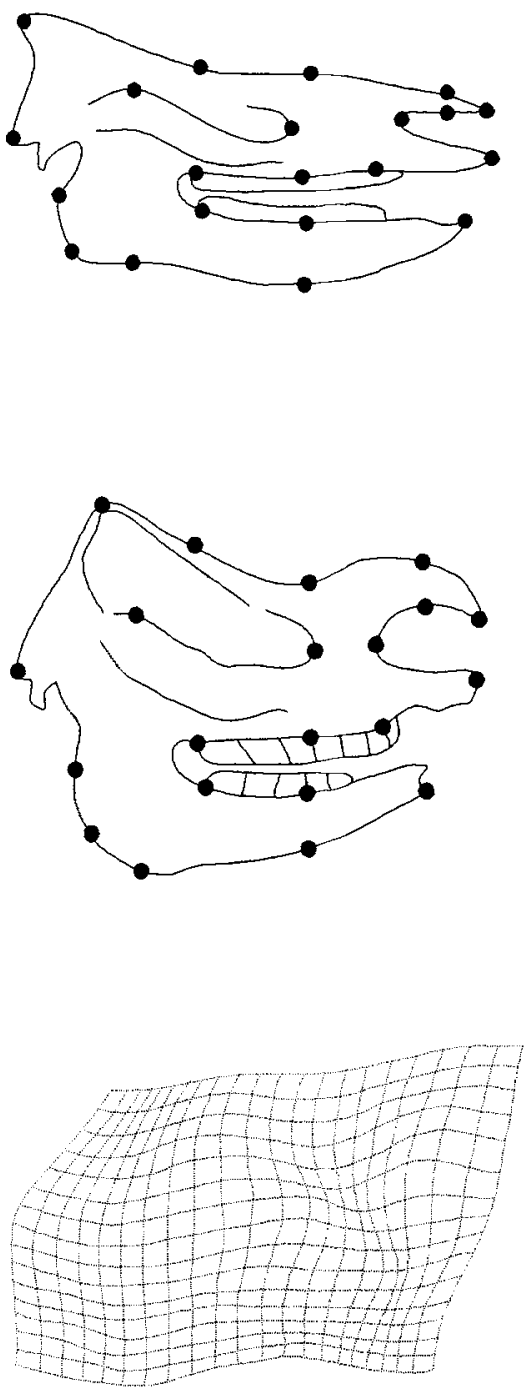

Fig. 5. Comparison of manual (Thompsonian) and computed (Thin-plate Splines) methods for the Cartesian transformation of Subhyracodon to Rhinoceros. (a) Replication of analysis by Colbert (1935). (top) Reference grid of squares is drawn over primitive specimen. (middle) Corresponding lines are drawn over derived specimen such that line-specimen relationships are maintained. (bottom) Resultant grid represents deformation of primitive to derived shape. (b) Comparable sets of landmarks are determined on primitive (top, Subhyracodon) and derived (middle, Rhinoceros) specimens. (bottom) Resultant grid represents the deformation necessary for a one-to-one superimposition of homologous landmarks (this result is the same as in Fig. 7b). 
particular distribution of landmarks across the shape as well as the way landmarks are initially fit before superimposition. Undoubtedly, these factors influence the nature of the resulting local deformations which may turn out to be counterintuitive in some cases. Thus, deepening of the skull, as observed in Colbert's grid, is not evident in the TPS grid, although some of the cells in the occipital region (upper left) are vertically elongated. These observations emphasize potential limitations of TPS results and the care which must be used in their interpretation.

The affine and non-affine components of the transformation of Subhyracodon to Rhinoceros were investigated. Because of the scale of this study (whole skull) and the various limitations on landmarks, the nonaffine deformations were not found to be individually useful and are not reported. These may be of interest in more localized studies of individual bones or small regions, as in Swiderski (1993). The global uniform affine component (Fig. 6) is particularly interesting because it accounts for most of the transformation from dolichocephaly (long shallow skull of Subhyracodon) to brachycephaly (short, deep skull of Rhinoceros). However, it is only a suggestion of the elegant global transformations of fish demonstrated by Thompson (1917). The relationship between shape change, as observed in such global geometries, and the evolution of developmental programs remains to be determined.

\section{TPS Transformations-Extant Genera}

Figure 7 shows the skull outlines of the four extant genera to which Subhyracodon was transformed using the landmarks discussed above. The transformation results for each will be discussed in approximate phylogenetic order (top to bottom).

Dicerorhinus (Figure 7a) is the most plesiomorphous living rhinoceros and this is

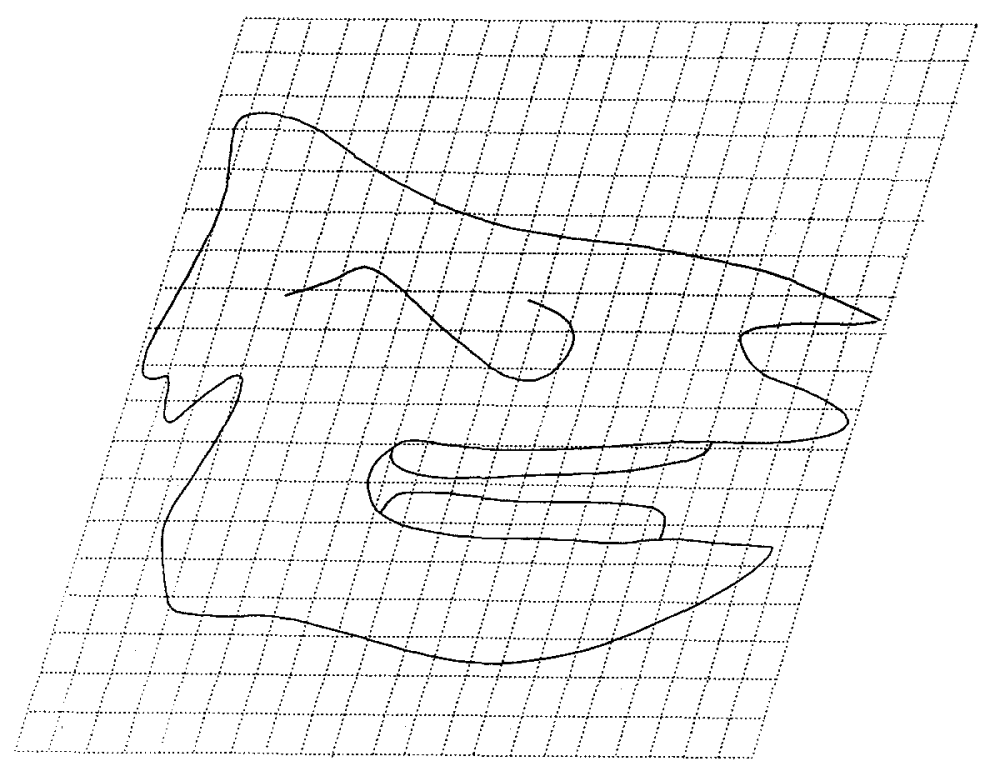

Fig. 6. Affine (global uniform) component of TPS factoring of Subhydracodon to Rhinoceros transformation. Outline represents deformed Subhyracodon. 
reflected in its skull shape and transformation grid. In overall shape, it is the most dolichocephalic of the living forms and thus is most like Subhyracodon (see Fig. 4). TPS emphasizes small changes: (1) some shortening of the orbitonasal distance, (2) some expansion of the nasals, and (3) a small amount of forward occipital rotation. The first two effects are seen in all of the extant forms. Expansion of the nasal region is correlated with the evolution of keratinous epidermal horns (Subhyracodon was hornless) and does not appear to have any other obvious functional significance.

Rhinoceros (Fig. 7b) represents a derived phylogenetic endpoint in terms of skull shape but is closely related to Dicerorhinus (closer than to the African taxa). The details of the TPS transformation to Rhinoceros are discussed in the preceding section where it is compared to Colbert's TT grid.

Diceros (Fig. 7c) appears to be the less derived of the two African genera from a morphological view, but it is nevertheless distinct from Subyracodon. Several aspects of its skull shape stand out in the TPS transformation. Expansion and elevation of the nasal region "pushes" the grid significantly upward such that the upper right corner of the grid is much higher than the upper left corner. This effect is contrary to the actual skull where the occiput remains higher than the nasals. However, by doing this, the grid emphasizes where significant change has occurred between the two forms. Another anterior feature shown is the reduction of the dentition associated with the premaxilla, producing lines which have become compressed together in that region. The occipital region of Diceros is little changed over Subhyracodon, with the tip of the occiput and the occipital condyle approximately in line vertically in both. What is emphasized posteriorly occurs in the region of the mandibular angle of Diceros. The rightward curvature of the vertical grid lines in the lower left corner suggests that the mandibular angle has shifted anteriorly, been reduced, or otherwise changed shape. Because the body of the mandible does not appear to be shifted, a relative reduction or shape change is indicated. Inspection of the mandibular angle in Subhyracodon shows it to be more squared while in Diceros it is more rounded. The grid therefore emphasizes this shape difference which may or may not represent a change in relative area. The mandible of Diceros, like that of Rhinoceros is deeper relative to its length than is that of Subhyracodon, but this is not reflected by TPS.

Ceratotherium (Fig. 7d) is similar to the other extant genera with respect to nasal enlargement, and similar to Diceros with respect to reduction of the anterior dentition. Two features of this transformation stand out from the others. The distortion of the grid lines near the upper middle of the grid shows the backward and upward shifting of the orbit relative to the tooth row (only slight in the other taxa). Most importantly is the posterior displacement of the occiput relative to the occipital condyle. As seen in the grid, the upper left corner is to the left of the lower left corner, an effect distinctly different from the other grids. The relatively small area of the mandibular angle (compare outline with Rhinoceros) is somewhat obscured by the displacement of the occiput, but it can be seen in the grid as a moderate compression of the vertical lines.

In summary, the TPS approach to Cartesian transformation emphasizes the following net changes between Subhyracodon and one or more of the extant genera: (1) enlargement and elevation of the nasal region, (2) changes in orbitonasal length and orbital position, (3) relative orientation of the occiput (forward in Rhinoceros, backward in Ceratotherium), and (4) shape and position of the mandibular angle (relative enlarge- 
a.
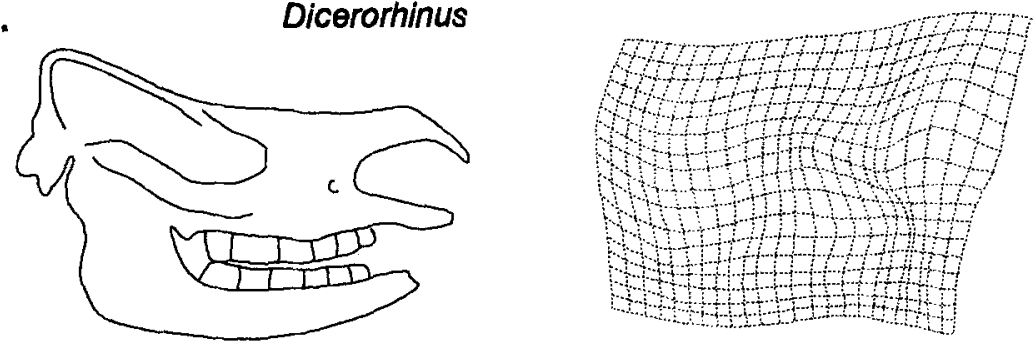

b.
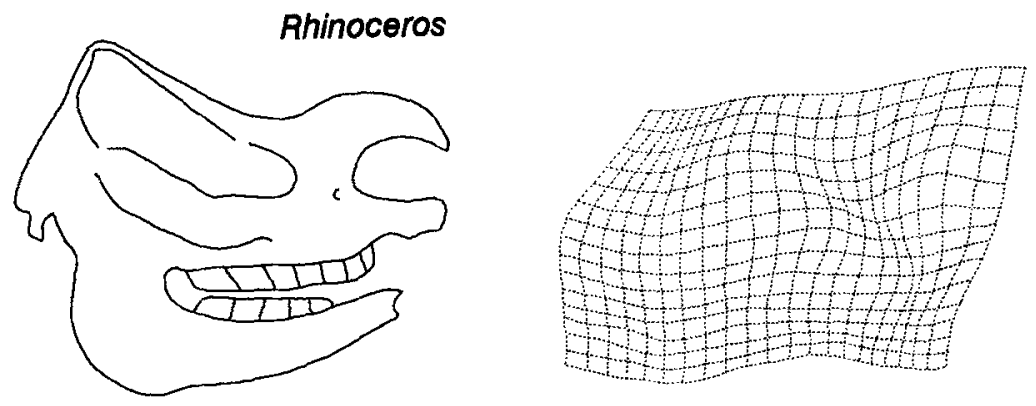

C.

Diceros
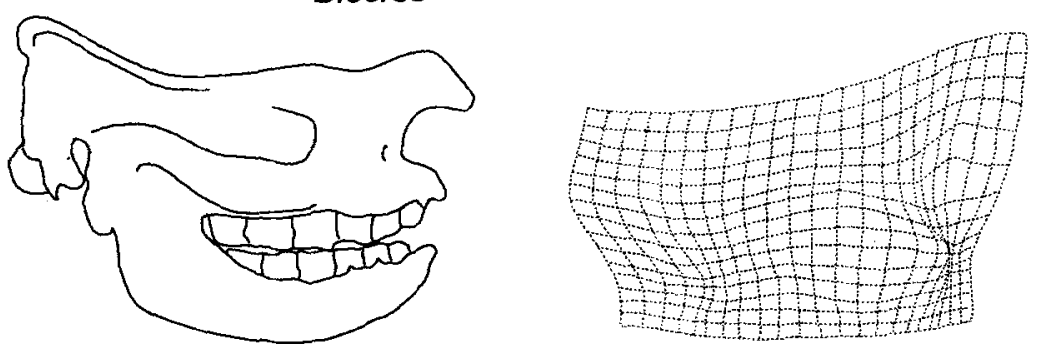

d.

Ceratotherium
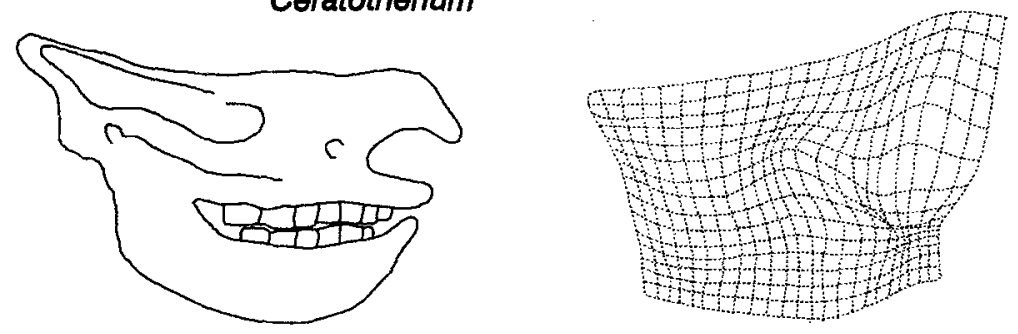

Fig. 7. Thin-plate Spline transformations from Subhyracodon to each of the extant rhinoceros genera. Specimen outlines (left, landmarks not shown) and corresponding deformation grids (right). (a) Dicerorhinus, (b) Rhinoceros (same transformation as in Fig. 5b, bottom), (c) Diceros, (d) Ceratotherium. 
ment in Rhinoceros and reduction in Ceratotherium). TPS did not emphasize relative differences along the dolichocephaly-brachycephaly spectrum or the actual shapes of the skulls. These results suggest that TPS should be viewed as supplementary and/or complimentary to the classic (visual) method.

\section{Functional Interpretations}

The rhinoceros skull, like all skulls, is a complex composite whose structure is determined by multifactorial influences. Natural selection sorts out the relative contributions of these influences to the functioning whole. Because most structures are multifunctional, they will tend to represent compromises between competing functions and mechanical requirements. Determining these intercorrelations among structures, functions, and mechanics is one of the goals of functional and comparative evolutionary morphology. Here, it is hypothesized that the differences in occipital orientation and mandibular angle size described above may be influenced, in addition to other factors, by the need to support a large mandible against gravity.

Figure 8 shows comparisons of Rhinoceros and Ceratotherium with Subhyracodon relative to common reference frames. It is clear in Fig. 8a that the posterior portion of the temporalis muscle in Ceratotherium has a greater posterior pull on the coronoid process, while in Rhinoceros it has a greater vertical pull. From this perspective, the differences would be attributable to differences in masticatory function (mandibular motion and occlusal forces), for example, in providing differential proportions of elevation versus retraction relative to the skull itself. Similarly, the differences of the mandibular angles would most likely reflect qualitative and/or quantitative differences in masseter function during mastication. Figure $8 \mathrm{~b}$ shows another potential effect of the two extremes in occiput position. All other things being equal, Rhinoceros can extend its head further. This correlates well with its habit of feeding on tall grasses (see below). Ceratotherium, on the other hand, is a grazer of low grasses and does not utilize head extension during feeding. Although these interpretations are reasonable given a common reference frame, it is of further interest to examine the role of functionally important head positions, especially with respect to gravity, on the skull shape differences.

The living rhinoceroses are megafauna and their mandibles accordingly contribute a substantial amount of weight to the skull. Because the mandible is a separate element suspended below the craniofacial skull, at least some aspects of its associated musculoskeletal morphology may reflect a weight bearing function. Further, the predominating position of the head will influence the details of the gravitational and countergravitational forces acting on the mandible. As large herbivores, rhinoceroses spend much of their daily activity schedule in foraging (Owen-Smith, 1988). Thus, forces acting on and within the skull while in the feeding position can be expected to be influential on skull shape. Subhyracodon, probably a browser, was small by comparison (Great Dane-sized) and the weight of its scaled-down mandible would be much less of a factor in the function of attached muscles. The evolution of large size in Diceros and Ceratotherium would require some adjustment to compensate for the scaling of mandibular weight.

Kurten (1968) briefly discussed head positions in Diceros, Ceratotherium, and Rhinoceros in the context of inferring feeding habits for Pleistocene fossil thinos. He explicitly correlated forward occipital position with head held up (Rhinoceros) but did not 

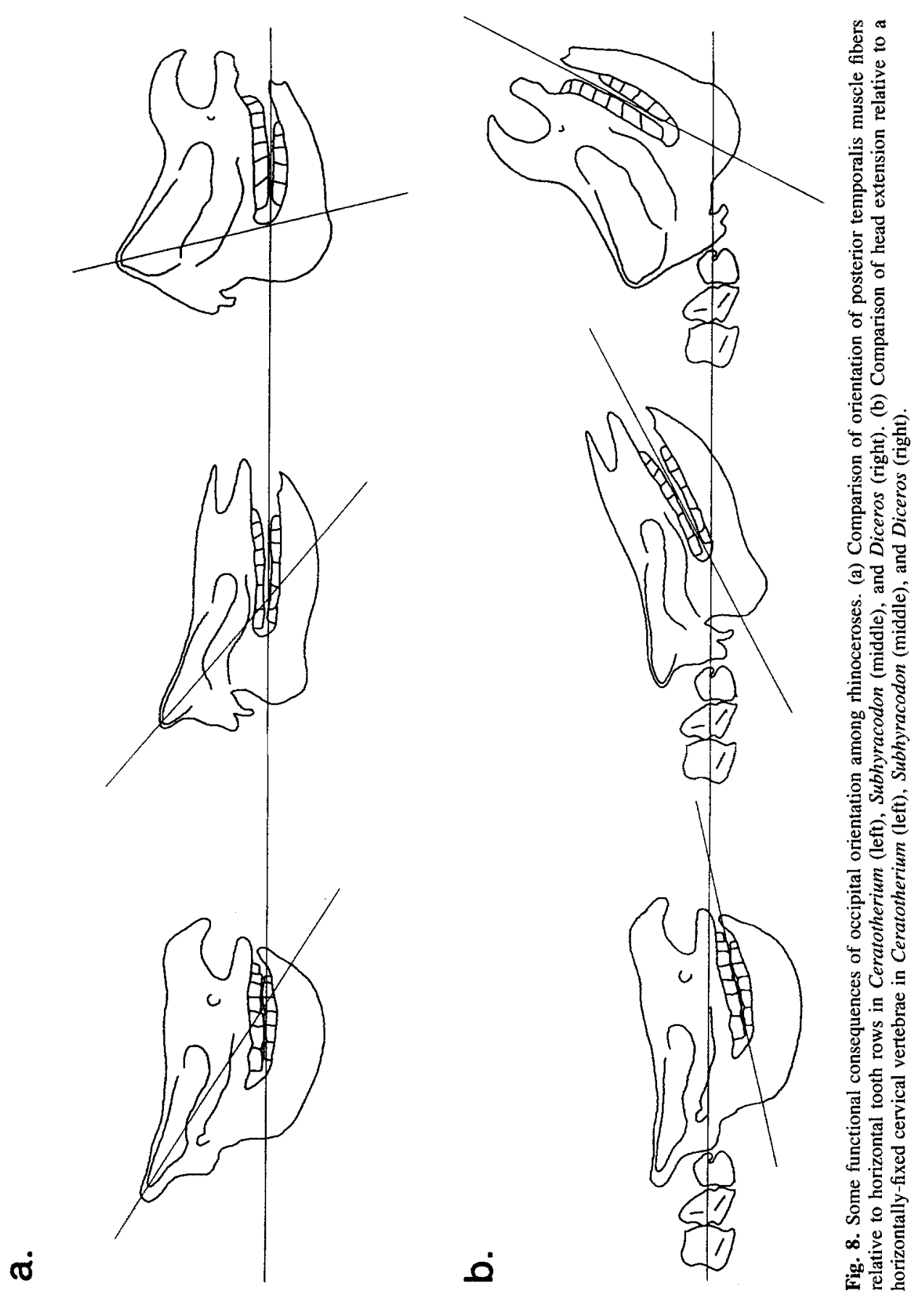
correlate head held down (Ceratotherium) or head held level (Diceros) with occipital positions. In a section headed "'general trends," Heissig (1989) stated that occipital plate orientation deviates in both directions from a right angle, and that the deviations reflect normal skull position which in turn is dependent on feeding position. Figure 9 shows an analysis of posterior temporalis and masseter muscle orientation relative to head position and gravity in Ceratotherium and Rhinoceros. The head position of Ceratotherium (Fig. $9 \mathrm{a}$, top) is shown in a lowered position as would be expected during grazing on short grass. Owen-Smith (1988) reported white rhino dietary grass height averaging $200 \mathrm{~mm}$ or less over the year. As shown, the head could be held higher or lower and thus represents an arbitrary, but reasonable, choice. Decomposition of the muscle force vectors (Fig. 9, bottom) into their rectangular components shows the relative pull of each muscle against gravity. In the lowered position of Ceratotherium, the posterior portion of the temporalis $\left(\mathrm{PT}_{\mathrm{C}}\right)$ has a large vertical (antigravity) component while the masseter $\left(\mathrm{M}_{\mathrm{C}}\right)$ has a small vertical component. In contrast, the head position of Rhinoceros (Fig. 8, top) is shown in a raised position, as would be expected during feeding on tall grasses. The predominant year-round dietary vegetation of Indian rhinos consists of tall cane-like grasses (Laurie, 1982; Owen-Smith, 1988). Again, the position shown is arbitrary but within the range of expected positions during feeding. For Rhinoceros (Fig. 9b, bottom), the force components of the posterior part of the temporalis $\left(\mathrm{PT}_{\mathrm{R}}\right)$ are approximately equal, while the pull of the masseter $\left(M_{R}\right)$ is entirely vertical.

a.

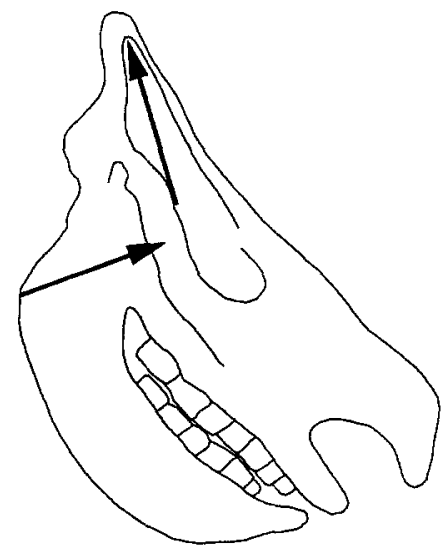

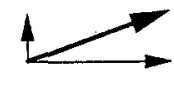

$\mathrm{PT}_{\mathbf{C}}$

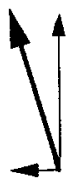

$\mathrm{M}_{\mathrm{C}}$ b.

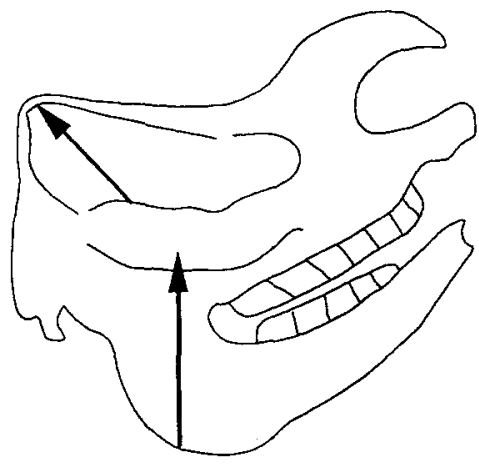

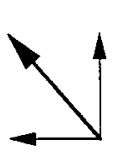

$\mathrm{PT}_{\mathbf{R}}$

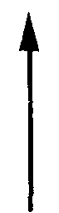

$M_{R}$

Fig. 9. Masticatory muscle orientations (lines of action) relative to feeding positions of the two extreme skull shapes. (a) Ceratotherium and (b) Rhinoceros. Thick artows: posterior temporalis (PT) and masseter (M) muscle orientations. Thin arrows: perpendicular force components for Ceratotherum $\left(\mathrm{PT}_{\mathrm{C}} ; \mathrm{M}_{\mathrm{C}}\right)$ and Rhinoceros $\left(\mathrm{PT}_{\mathrm{R}} ; \mathrm{M}_{\mathrm{R}}\right.$ ) muscles. 
The occipital and mandibular angle differences between the two derived taxa Ceratotherium and Rhinoceros may be partially explained by the force components of the two masticatory muscles discussed. In Ceratotherium, the masseter does little work supporting the mandible against gravity (in a still lower position, the masseter would provide even less and the posterior temporalis fibers would provide more support). The masseter's size, therefore, should be correlated mostly with swinging the mass of the mandible forward and providing bite force. The Rhinoceros masseter, in addition to overcoming masseter inertia and providing bite force, must provide extra force to counteract gravity. Thus, the relatively larger mandibular angle of Rhinoceros may reflect a larger masseter required to provide the extra force during feeding (by analogy, it requires more force to lift a refrigerator than to push one). The posterior temporalis fibers, acting on the same bony element, should be expected to correlate with the masseters to some degree, if support is a significant function. The vertical component of $\mathrm{PT}_{\mathrm{C}}$, for example, appears to compensate for a lack of that component in $\mathrm{M}_{\mathrm{C}}$. It may be hypothesized that occipital position in Ceratotherium (i.e., backwardly-rotated relative to the ancestral condition) is at least partly explained by the need for this compensation in the lowered feeding position. Of course, relative to the skull itself, it is providing a retractive force on the mandible. It must also be remembered that anterior fibers of the temporalis play a role but are not associated here with observed morphological differences. The $\mathrm{PT}_{\mathrm{R}}$ has less of its pull directed vertically but is still significantly synergistic against gravity with $\mathbf{M}_{R}$ (anterior temporalis fibers, not shown, must also be pulling against gravity). The horizontal component of $\mathrm{PT}_{\mathrm{R}}$ is providing retraction. These observations suggest that the forward rotation of the occiput in Rhinoceros has helped maintain a vertical component to the action of posterior temporalis fibers in a raised feeding position.

In summary, evolved shape differences in the posterior skull regions of the two most divergent extant rhinoceroses are consistent with a role for the masticatory muscles in supporting large mandibles in the two different feeding positions.

\section{DISCUSSION}

The evolution of large size in terrestrial vertebrates is accompanied by musculoskeletal changes which can be correlated with mechanical needs. For example, the limbs of large animals generally have greater relative diameter and decreased joint angulation (graviportal limbs) than smaller animals (Alexander, 1985). Mechanically, the linear limb elements become progressively dominated by the need for columnar support of a volumetric mass (presumably in a grossly compressive environment). Within a skull, it is predictable that increasing mandible mass would proportionately increase the need for suspensory support (presumably, in a grossly tensional environment). The increasing need for suspension would require compromises with other functions of the masseter and temporalis (Smith, 1993). The results of two-dimensional shape analysis in this paper, correlated with direction of muscle pull and corresponding bony features, suggest that mandible suspension has evolved as an important function in the large extant rhinoceros skulls. Further, the function is tracked in two divergent genera with different feeding postures. Thus, the rhinoceroses may be an example where a relatively simple mechanical requirement has had a strong influence on skull shape, and can be relatively simply correlated with feeding behavior. It is interesting to speculate how the muscle fiber types 
and motor units might be apportioned, because the support must be a continuous function of the muscles in contrast to intermittant, heterogeneous chewing functions (Herring et al., 1979).

In the light of current research interest and advances in the evolution and development of the vertebrate skull (see Hanken and Hall, 1993a,b), and the predominance of cladistic character analysis in systematics and phylogeny reconstruction (Maddison, 1994; Padian et al., 1994), it is useful to briefly discuss the present results in those two contexts.

With respect to rhinoceros skull ontogenetic evolution, some fundamental questions include: when do the generic or specific shape differences appear; what are the proportions of genetic versus epigenetic factors on shape; what are the epigenetic factors (e.g., what is the tensional/compressional "history" of the skull); and how does behavioral ontogeny correlate with morphological ontogeny? In terms of postnatal epigenetic mechanical effects, the suckling offspring of both species probably have approximately the same head orientation at least some of the time. It is especially important to determine the relationship between the time course of weaning to vegetation and differences in skull shape during this period when they begin to behaviorally diverge and the skull and mandible become more massive. Unfortunately, descriptive and experimental rhinoceros embryology is nonexistent. Some inferences can be made based on the craniological literature (Emerson and Bramble, 1993; Herring, 1993; Russell and Thomason, 1993; Smith, 1993).

First, occiput orientation (shape) does not seem to have been developmentally or evolutionarily constrained. Rather, orientation evolved in opposite directions according to functional-mechanical needs. This would not be the case, for example, if brain growth/ evolution were significantly controlling occiput shape (a study of rhinoceros endocranial volume and shape would be interesting). Next, it is reasonable to assume that at least some of the occiput shape and mandible angle size are regulated by proximate effects of associated muscles (Herring, 1993). Groves (1982) noted examples of plastic deformation in the skulls of captive rhinos, usually feeding under unnatural conditions. Finally, because the occiput serves multiple functions (e.g., for nuchal and temporal musculature attachments and for brain encasement), it must represent a compromise between multiple functional matrices (Moss, 1968). In the view of Moss, a functional matrix evolves and the skeleton responds. For this example, evolution of different behavioral orientations changes muscle needs which in turn provides the environment (matrix) within which the occiput "responds." Interestingly, the forward inclination of the occiput in Rhinoceros seems to be an example of synergism among functional matrices, because it allows greater head extension ("nuchal matrix") as well as support ("mandible matrix") at the same time.

With respect to systematics and phylogeny reconstruction, it may be asked: to what extent have the occipital and mandibular angle differences between Rhinoceros and Ceratotherium been correlated with, or used as, taxonomic characters (especially in more recent cladistic analyses)? Skull shape is clearly an important aspect of what these two divergent species are and what they do. And clearly, the occipital differences are derived characteristics (autapomorphies) distinguishing the two genera from Subhyracodon and from each other. However, it might be argued that any rhino lineage achieving large size and feeding in the same way might convergently have similar features. The black 
rhino (Diceros), for example, is known to be a closer relative of Ceratotherium (Prothero et al., 1986; Morales and Melnick, 1994), yet its occipital orientation is intermediate to slightly-rotated forward, consistent with its characterization as a "horizontal" browser (Kurten, 1968). It is well known that gross features resulting from mechanical causes, such as graviportality or fusiform shape, are not necessarily useful for determining monophyly because of convergences. The relationship between cladistics and morphometrics has only recently begun to be investigated as a result of the maturing of the former and the advances of the latter (e.g., Warheit, 1992; David and Laurin, 1996).

Differences of mandibular angle among rhinoceros taxa have not been important systematically. Differences of occipital shape (position) have been cited historically, but not universally. Cuvier (1834) was probably the first to note occipital differences (implied in Flower, 1876). Flower cited occipital crest position as a feature separating the Indian and Javan rhinos (Rhinoceros) from the Sumatran rhino (Dicerorhinus). Osborn (1903) mentioned the "high forward-sloping occiput" in Rhinoceros but not the opposite condition in Ceratotherium. Pocock (1945) discussed the forward inclination of the occiput in Rhinoceros as a well known difference separating it from Didermocerus [Dicerorhinus]. Meester and Setzer (1971) relied heavily on the backward position of the occiput as a feature distinguishing Ceratotherium from Diceros. In an analysis of the interrelationships of the living genera, Groves (1983) used occipital crest orientation to differentiate the Asian from African rhinos. His implication that the occiput slopes backward in Diceros is contradicted by the Cartesian results. The most recent and comprehensive character analysis of rhinoceroses is that of Prothero et al. (1986) which provides a phylogeny based on shared derived characters. Neither occipital nor mandibular angle features are cited as characters distinguishing the black and white rhinos (subtribe Dicerotina) from each other, or distinguishing them from Rhinoceros (subtribe Rhinocerotina). Each subtribe contains a less derived member (Diceros and Dicerorhinus, respectively). Thus, the shape differences have occurred relatively recently, and entirely within the respective generic lineages. It seems that behavioral-physiological canalization for vegetation preferences in the two lineages determined which direction skull shape would adapt to accommodate a massive mandible in different orientations.

This paper presents a "postcladistic" analysis of shape change in two lineages of a mammalian phylogeny with fairly high confidence. Analyses of shape change in such cases should be valuable in understanding the causes and consequences of character evolution within the phylogenies. Further craniological analyses of Rhinoceros and Ceratotherium may provide an opportunity for some integration of evolution, biomechanics, development, behavior, ecology, systematics, and phylogeny within an ancient mammalian clade. This I hope will stimulate research on the rhinoceroses, and in turn, broaden and deepen interest in conserving these deserving representatives of the Age of Mammals.

\section{ACKNOWLEDGMENTS}

A prelíminary version of this paper was reviewed by Gene H. Albrecht, Department of Cell and Neurobiology, University of Southern California. Thanks are extended to Dr. Albrecht and three anonymous reviewers for their helpful comments. 


\section{REFERENCES}

Alexander, R. M. (1985). Body support, scaling, and allometry. In: Functional Vertebrate Morphology, M. Hildebrand, D. M. Bramble, K. F. Liem, and D. B. Wake, eds., pp. 26-37, Harvard University Press, Cambridge, MA.

Bookstein, F. L. (1989). Principal warps: Thin-plate splines and the decomposition of deformations. I.E.E.E. Trans. Pattern Anal. Mach. Intell. 11: 567-585.

Bookstein, F. L. (1990). Introduction to methods for landmark data. In: Proceedings of the Michigan Morphometrics Workshop, F. J. Rohlf and F. L. Bookstein, eds., Special Publication No. 2, University of Michigan Museum of Zoology, Ann Arbor.

Bookstein, F. L. (1992). Morphometric Tools for Landmark Data: Geometry and Biology: Cambridge University Press, New York.

Colbert, E. H. (1935). Siwalik mammals in the American Museum of Natural History. Trans. Am. Phil. Soc. Ser. 2 26: 177-214.

Cuvier, G. (1834). Reserches sur les Ossemens Fossiles Vol. 3. Paris.

David, B., and Laurin, B. (1996). Morphometrics and cladistics: measuring phylogeny in the sea urchin Echinocardium. Evolution 50: 348-359.

Emerson, S. B., and Bramble, D. M. (1993). Scaling, allometry, and skull design. In: The Skull Vol. 3. Functional and Evolutionary Mechanisms, J. Hanken and B. K. Hall, eds., pp. 384-421, University of Chicago Press, Chicago.

Flower, H. F. (1876). On some cranial and dental characters of the existing species of rhinoceros. Proceed. Roy. Soc., Lond. 1876: 443-457.

Groves, C. (1982). The skulls of Asian rhinoceroses: Wild and captive. Zoo Biol. 1: 251-261.

Groves, C. (1983). Phylogeny of the living species of thinoceros. Z.f. zool. Syt. u. Evolutionsforschung 21: 293-313.

Hanken, J., and Hull, B. K., eds. (1993a). The Skull Vol. 1. Development, University of Chicago Press, Chicago.

Hanken, J., and Hall, B. K., eds. (1993b). The Skull Vol. 3. Functional and Evolutionary Mechanisms, University of Chicago Press, Chicago.

Heissig, K. (1989). The Rhinocerotidae. In: The Evolution of Perissodactyls, D. R. Prothero and R. M. Schoch, eds., pp. 227-276, Oxford University Press, New York.

Herring, S. (1993). Functional morphology of mammalian mastication. Am. Zool. 33: 289-299.

Herring, S., Grimm, A. F., and Grimm, B. R. (1979). Functional heterogeneity in a multipennate muscle. Am. J. Anat. 154: 563-575.

Hildebrand, M., Bramble, D. M., Liem, K. F., and Wake, D. B., eds. (1985). Functional Vertebrate Morphology, Harvard University Press, Cambridge, MA.

Kurten, B. (1968). Pleistocene Mammals of Europe, Aldine Publishing, Chicago.

Laurie, A. (1982). Behavioural ecology of the greater one-horned rhinoceros (Rhinoceros unicornis). J. Zool. Lond. 196: 307-341.

Lotus (1993). Freelance Graphics Release 4.0 for DOS. Lotus Development Corporation. Cambridge, MA.

Maddison, D. R. (1994). Phylogenetic methods for inferring the evolutionary history and processes of change in discretely valued characters. Annu. Rev. Entomol. 39: 267-292.

Meester, J., and Setzer, H. W., eds. (1971). The Mammals of Africa: An ID Manual. Smithsonian Institution Press, Washington, D.C.

Morales, J. C., and Melnick, D. J. (1994). Molecular systematics of the living rhinoceros. Molec. Phylogen Evol. 3(2): 128-134.

Moss, M. (1968). A theoretical analysis of the functional matrix. Acta Biotheoretica 18: 194-202.

Osborn, H. F. (1903). The extinct rhinoceroses. Memoirs. Am. Mus. Nat. Hist. 1: 75-164.

Owen-Smith, R. N. (1988). Megaherbivores. The Influence of Very Large Body Size on Ecology, Cambridge University Press, Cambridge, England.

Padian, K., Lindberg, D. R., and Polly, P. D. (1994). Cladistics and the fossil record: The uses of history. Annu. Rev. Earth Planet Sci. 22: 63-91.

Pizzaz Plus (1990). Application Technologies, Inc., Version 2.0.

Pocock, R. I. (1945). Some cranial and dental characters of the existing species of Asiatic rhinoceroses. Proc. Zool. Soc. Lond. 114: 437-450.

Prothero, D. R., and Schoch, R. M., eds. (1989). The Evolution of Perissodactyls, Oxford University Press, New York.

Prothero, D. R., Manning, E., and Hanson, C. B. (1986). The phylogeny of the Rhinocerotoidea (Mammalia, Perissodactyla). Zool. J. Linn. Soc. 87: 341-366.

Prothero, D. R., Guerin, C., and Manning, E. (1989). The history of the Rhinocerotoidea. In: The Evolution of Perissodactyls, D. R. Prothero and R. M. Schoch, eds., pp. 321-340, Oxford University Press, New York. 
Rohlf, F. J. (1990a). Rotational fit (procrustes) methods. In: Proceedings of the Michigan Morphometrics Workshop, F. J. Rohlf, and F. L. Bookstein, eds., pp. 227-236, Special Publication No. 2, University of Michigan Museum of Zoology, Ann Arbor.

Rohlf, F. J. (1990b). Thin-Plate Splines, Version 11/8/90.

Rohlf, F. J., and Bookstein, F. L., eds. (1990). Proceedings of the Michigan Morphometrics Workshop, Special Publication No. 2, University of Michigan Museum of Zoology, Ann Arbor.

Russell, A. P., and Thomason, J. J. (1993). Mechanical analysis of the mammalian head skeleton. In: The Skull Vol. 3. Functional and Evolutionary Mechanisms, J. Hanken and B. K. Hall, eds., pp. 345-383, University of Chicago Press, Chicago.

Simpson, G. G., Roe, A., and Lewontin, R. C. (1960). Quantitative Zoology, Rev. Ed. Harcourt, Brace, and Company, New York.

Slice, D. E. (1990). DS-Digit: Basic Digitizing Software, Department of Ecology and Evolution, State University of New York at Stony Brook, Stony Brook, New York.

Smith, K. K. (1993). The form of the feeding apparatus in terrestrial vertebrates: Studies of adaptation and constraint. In: The Skull Vol. 3. Functional and Evolutionary Mechanisms, J. Hanken and B. K. Hall, eds, pp. 150-196, University of Chicago Press, Chicago.

Swiderski, D. L. (1993). Morphological evolution of the scapula in tree squirrels, chipmunks, and ground squirrels (Sciuridae): An analysis using Thin-plate Splines. Evolution 47: 1854-1873.

Thompson, D. W. (1917). On Growth and Form. Cambridge University Press, London.

Warheit, K. (1992). The role of morphometrics and cladistics in the taxonomy of fossils: A paleoornithological example. Syst. Biol. 41: 345-369. 